\title{
Perfil sociodemográfico das mulheres que realizaram laqueadura tubária: uma revisão integrativa da literatura
}

Sociodemographic profile of women who underwent tubal ligation: an integrative literature review

Perfil sociodemográfico de mujeres sometidas a ligadura de trompas: revisión integradora de la

\section{Resumo}

A laqueadura tubária consiste na oclusão mecânica ou ressecção parcial das trompas de Falópio, ao mesmo tempo em que a junção dos gametas não consiga ocorrer, eliminando as probabilidades da fecundação. Esse método está entre a preferência de casais, quando optam por uma prática contraceptiva, tanto em países desenvolvidos como naqueles em desenvolvimento. O presente estudo baseia-se na seguinte pergunta norteadora: Qual o perfil sociodemográfico das mulheres que realizaram laqueadura? Diante disso, o objetivo do presente trabalho consiste em analisar o perfil sociodemográfico das mulheres que realizaram laqueadura por meio de um estudo de revisão de literatura do tipo Integrativa. Trata-se de uma revisão, por meio de produções científicas da base de dados: Literatura Latino-Americana em Ciências de Saúde - LILACS, Scientific Eletronic Library Online - SCIELO, Biblioteca Virtual em Saúde - BVS, dos últimos 5 anos, ou seja, de 2015 a 2020. Entre as mulheres com 3 ou mais filhos, a laqueadura foi a prática mais frequente, embora tenha sido superada pelo uso de hormonais injetáveis no grupo etário de 20 a 29 anos. Portanto, essa pesquisa abriu novas possibilidades para aprofundar os conhecimentos a respeito da laqueadura tubária e das variáveis ligadas as mulheres que escolhem esse procedimento. Essa realidade evidencia a necessidade de implantação de medidas de educação em saúde bem como de estratégias focadas nesse público, pois arrependimentos e consequências fazem parte desse processo, como foram pontuadas nos estudos selecionados.

Palavras-chave: Laqueadura; Arrependimento; Perfil sociodemográfico.

\begin{abstract}
Tubal ligation consists of mechanical occlusion or partial resection of the fallopian tubes, at the same time that the junction of the gametes cannot occur, eliminating the chances of fertilization. This method is among the preference of couples when they opt for contraceptive practice, both in developed and developing countries. This study is based on the following guiding question: What is the socio-demographic profile of women who underwent tubal ligation? Therefore, the aim of this study is to analyze the socio-demographic profile of women who underwent tubal ligation through an integrative literature review study. This is a review, through scientific productions from the database: Latin American Literature in Health Sciences - LILACS, Scientific Electronic Library Online - SCIELO, Virtual Health
\end{abstract}


Library - VHL, of the last 5 years, that is, from 2015 to 2020. Among women with 3 or more children, tubal ligation was the most frequent practice, although it was surpassed by the use of injectable hormones in the 20 to 29 age group. Therefore, this research opened new possibilities to deepen the knowledge about tubal ligation and the variables related to women who choose this procedure. This reality highlights the need to implement health education measures as well as strategies focused on this audience, as regrets and consequences are part of this process, as highlighted in the selected studies.

Keywords: Tubal ligation; Repentance; Sociodemographic profile.

\section{Resumen}

La ligadura de trompas consiste en la oclusión mecánica o resección parcial de las trompas de Falopio, al mismo tiempo que no se puede producir la unión de los gametos, eliminando las posibilidades de fecundación. Este método se encuentra entre la preferencia de las parejas cuando optan por prácticas anticonceptivas, tanto en países desarrollados como en vías de desarrollo. Este estudio se basa en la siguiente pregunta orientadora: ¿Cuál es el perfil sociodemográfico de las mujeres que se sometieron a ligadura de trompas? Por tanto, el objetivo de este estudio es analizar el perfil sociodemográfico de las mujeres sometidas a ligadura de trompas mediante un estudio de revisión integradora de la literatura. Se trata de una revisión, a través de producciones científicas de la base de datos: Literatura Latinoamericana en Ciencias de la Salud - LILACS, Biblioteca Electrónica Científica Online - SCIELO, Biblioteca Virtual en Salud - BVS, de los últimos 5 años, es decir, de 2015 a 2020. Entre mujeres con 3 o más hijos, la ligadura de trompas fue la práctica más frecuente, aunque fue superada por el uso de hormonas inyectables en el grupo de edad de 20 a 29 años. Por tanto, esta investigación abrió nuevas posibilidades para profundizar en el conocimiento sobre la ligadura de trompas y las variables relacionadas con las mujeres que optan por este procedimiento. Esta realidad pone de relieve la necesidad de implementar medidas de educación para la salud y estrategias enfocadas a esta audiencia, ya que el lamento y las consecuencias son parte de este proceso, como se destaca en los estudios seleccionados.

Palabras clave: Ligadura de trompas; Arrepentimiento; Perfil sociodemográfico.

\section{Introdução}

A concepção de saúde da mulher vincula-se à maternidade e a criança, por décadas essas ações dos governos do Brasil com relação à saúde pública voltada para a mulher estavam unicamente focada nesse sentido, que perdurou até a década de 1980, onde através de programas desenvolvidos com o objetivo de visar o controle da natalidade, entrou como política de controle populacional (Bezerra et al, 2018).

A partir do Movimento Feminista, se dá o processo de conquistas das mulheres no espaço institucional, com ações de políticas públicas voltadas a inclusão das mulheres em diferentes setores na sociedade, que vão desde a participação delas na política, em espaços de trabalho público, à busca da melhoria da saúde das diferentes formas de conhecer o seu corpo e aborda questões que fazem parte do cotidiano das mulheres, como aborto, sexualidade, direito de ter ou não filho, violência doméstica e muitos outros, com isso abrindo possibilidades para que milhares questionassem as relações de gênero, suas relações não igualitárias com seus maridos, famílias e comunidades (Marcondes, 2018).

Com isso os vários planejamentos de ações de saúde, tinham um objetivo de promover a melhoria na condição de vida, a igualdade e os direitos de cidadania da mulher. A manutenção da boa saúde da mulher exige uma série de cuidados e atitudes preventivas, pois a mulher não está apenas associada no processo de reprodução biológica, mas dentro de uma assistência geral de saúde como uma cidadã detentora de direitos sexuais, culturais, socioeconômicos bem como sendo incluídos nas ações educativas, preventivas, de diagnóstico, tratamento e recuperação preconizando a assistência à mulher em clínica ginecológica, dentre estes o pré-natal, parto, puerpério e prevenção do câncer do colo uterino (Bezerra et al, 2018; Teodoro et al., 2020).

Diante do exposto, existe um ponto a se abordar diretamente, "A autonomia da mulher frente a laqueadura nos dias atuais e a preservação dos seus direitos". Apesar dos inúmeros avanços com os métodos de contraceptivos, não existe nenhum método $100 \%$ eficaz, uma vez que todos eles apresentam alguma probabilidade de falhar, e que nenhum é melhor que o outro, pois na verdade, o que existe, são apenas as vantagens e desvantagens de cada método (Brasil, 2013). 
O planejamento da vida sexual e reprodutiva constitui-se numa questão de cidadania, como o reconhecimento e a garantia dos direitos reprodutivos e sexuais, por meio das informações, da escolha livre e informada dos métodos contraceptivos que o casal deseja utilizar-se, respeitando os princípios da dignidade da pessoa (Fernandes, 2020).

Portanto, os direitos reprodutivos estão relacionados aos direitos das mulheres e a autonomia e igualdade de gênero. No entanto, existem muitas controvérsias, pois muitas vezes, esses direitos apresentam raízes na tradição, na cultura e na religião (Gonçalves, 2017). Diante disso, o interesse em realizar essa pesquisa nasceu de experiências profissionais e pessoais vividas no cotidiano da enfermagem, haja visto, os estágios também surgem como excelente oportunidade para vivenciar situações profissionais que acabam impulsionando para escolhas de temas de trabalhos ou até mesmo de especializações futuras

O objetivo do presente estudo consiste em analisar o perfil sociodemográfico das mulheres que realizaram laqueadura por meio de um estudo de revisão de literatura do tipo Narrativa. E os específicos são: identificar o perfil sociodemográfico de mulher que realizaram laqueadura; analisar a importância da laqueadura e discorrer sobre os riscos e benefícios da laqueadura. E assim, contribuir com os interessados no tema, profissionais, comunidade em geral e gestores, a fim de fornecer subsídios para elaboração ou implementações, visando beneficiar a saúde da mulher.

\section{Metodologia}

\subsection{Tipo de pesquisa}

A metodologia ajuda o pesquisador responder os questionamentos a respeito da temática levantada, nesse caso, o "Perfil sociodemográfico das mulheres que realizaram laqueadura", em observância aos direitos da mulher, ou seja, como descobrir o número de mulheres laqueadas; quem são essas mulheres; casadas ou solteiras e se houve complicações em partos anteriores.

O método é o caminho a ser percorrido para que o objetivo da pesquisa seja alcançado. Parra Lakatos e Marconi (2010) o método "é o caminho a ser trilhado pelos pesquisadores na busca do conhecimento": é o traçado das etapas fundamentais da pesquisa.

O presente trabalho consiste em uma pesquisa bibliográfica desenvolvida através do método da Pesquisa Integrativa. A pesquisa bibliográfica é fundamental nas práticas de investigações, pois através dos registros publicados o investigador tem a oportunidade de encontrar respostas de lacunas existentes (Gil, 2008).

Por outro lado, segundo Bruner (2002, p. 46), autor utilizado para o embasamento teórico desta pesquisa, afirma que: "uma narrativa é composta por uma sequência singular de eventos, estados mentais, ocorrências envolvendo seres humanos como personagens ou autores" e acrescenta, mais à frente que "ela ode ser "real" ou "imaginária" sem perder seu poder como história".

\subsection{Pergunta norteadora}

Qual o perfil sociodemográfico das mulheres que realizaram laqueadura?

\subsection{Busca na literatura}

Para coletar os dados foi realizada uma investigação nas bases de dados: Biblioteca Virtual em Saúde (BVS), Literatura Latino-Americana em Ciências de Saúde (LILACS), Base de Dados Bibliográficos Especializada na Área de Enfermagem e Scientific Eletronic Library Online (SCIELO). Sendo assim 10 artigos foram selecionados. 
A investigação da literatura foi realizada por meio do cruzamento das palavras-chave: Laqueadura; Saúde da Mulher; Parto e Complicações. Os Descritores em Ciências da Saúde (DECS) são: Esterilização Tubária; Assistência Integral à Saúde da Mulher; Apresentação no Trabalho de Parto e Declínio Pós-Opertório.

\subsection{Critérios de elegibilidade}

Os critérios de inclusão utilizados para a seleção da amostra foram: artigos publicados no período de 2015 a 2020 , com temas voltados para o perfil sociodemográfico das mulheres que realizaram laqueadura. Foram excluídos durante a busca: produção duplicada, editoriais, cartas ao editor, boletins epidemiológicos, dissertação, monografia, artigo de revisão.

\subsection{Protocolo de triagem dos artigos}

A avaliação crítica dos estudos selecionados consistiu-se na leitura na íntegra dos mesmos (prisma), Figura 1, seguida da elaboração de quadros contendo ano de publicação e sujeitos do estudo.

Figura 1. Trajetória artigos encontrados, 2021.

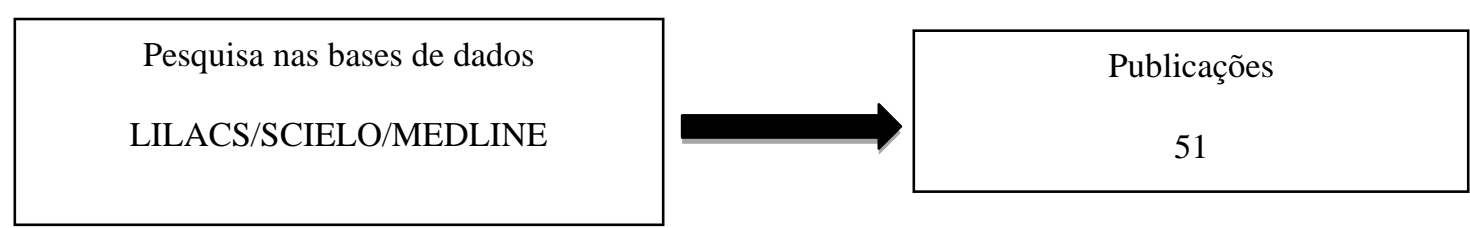

Aplicação dos critérios de exclusão

Publicações em idiomas não selecionados: 12

Publicações em documentos diferentes ao formato artigo: 08

Publicações sem o texto completo online: 08

Publicações que não abordam discussões pertinentes às questões norteadoras: 17

\begin{tabular}{|l|l|}
\hline Total de artigos selecionados & $\begin{array}{l}\text { Biblioteca Virtual em Saúde (BVS), Literatura } \\
\text { Latino-Americana em Ciências de Saúde } \\
\text { (LILACS), Base de Dados Bibliográficos } \\
\text { Especializada na Área de Enfermagem; Scientific } \\
\text { Eletronic Library Online (SCIELO) }=06\end{array}$ \\
\hline
\end{tabular}

Fonte: Autoria própria (2021).

\section{Resultados}

A amostra final desta revisão foi constituída por 06 artigos científicos, selecionados pelos critérios de inclusão previamente estabelecidos. O Quadro 1 representa as especificações de cada um dos artigos, distribuídos, segundo: ano; periódico; nome dos autores e título. 
Quadro 1. Relação dos estudos selecionados quanto ao ano, periódico, autores e título entre 2015 e 2020 . Imperatriz-MA 2021.

\begin{tabular}{|c|c|c|c|}
\hline $\begin{array}{l}\text { AUTOR/ANO DE } \\
\text { PUBLICAÇÃO }\end{array}$ & TÍTULO & AMOSTRA & $\begin{array}{l}\text { ABORDAGEM } \\
\text { METODOLÓGICA }\end{array}$ \\
\hline $\begin{array}{l}\text { Da Fonseca Bezerra, J. et } \\
\text { al } 2018\end{array}$ & $\begin{array}{l}\text { Assistência à mulher frente à violência sexual e } \\
\text { políticas públicas de saúde: revisão integrativa }\end{array}$ & 21 artigos & $\begin{array}{l}\text { Estudo } \\
\text { de revisão integrativa }\end{array}$ \\
\hline $\begin{array}{l}\text { Brandão, E. R., Pimentel, } \\
\text { A. C. de L., } 2020\end{array}$ & $\begin{array}{l}\text { Essure no Brasil: desvendando sentidos e usos sociais } \\
\text { de um dispositivo biomédico que prometia esterilizar } \\
\text { mulheres }\end{array}$ & $\begin{array}{l}\text { Dezembro de } 2018 \\
\text { a maio de } 2019\end{array}$ & Pesquisa documental \\
\hline $\begin{array}{l}\text { Lago, T. Di G. do, et al, } \\
2020\end{array}$ & $\begin{array}{l}\text { Diferenciais da prática contraceptiva no Município de } \\
\text { São Paulo, Brasil: resultados do inquérito populacional } \\
\text { Ouvindo Mulheres }\end{array}$ & 4 mil mulheres & Pesquisa de campo \\
\hline $\begin{array}{l}\text { Lima, I. C. V. de, et al, } \\
2017\end{array}$ & $\begin{array}{l}\text { Aspectos reprodutivos e conhecimento sobre } \\
\text { planejamento familiar de mulheres com Síndrome da } \\
\text { Imunodeficiência Adquirida }\end{array}$ & 102 pacientes & $\begin{array}{l}\text { Estudo transversal, descritivo } \\
\text { e quantitativo }\end{array}$ \\
\hline $\begin{array}{l}\text { Sanguinete, T. A. A., } \\
2020\end{array}$ & $\begin{array}{l}\text { Perfil das mulheres que realizaram laqueadura em uma } \\
\text { maternidade de referência do Alto do Jequitinhonha - } \\
\text { MG }\end{array}$ & & $\begin{array}{l}\text { Estudo quantitativo, } \\
\text { retrospectivo e documental }\end{array}$ \\
\hline $\begin{array}{l}\text { Spigolon, D. N., et al, } \\
2020\end{array}$ & $\begin{array}{l}\text { Percepções das gestantes quanto à escolha da via de } \\
\text { parto }\end{array}$ & 20 gestantes & $\begin{array}{l}\text { Estudo descritivo com } \\
\text { abordagem qualitativa }\end{array}$ \\
\hline
\end{tabular}

Fonte: Autoria própria (2021).

Foi realizada leitura analítica dos artigos selecionados que possibilitou a organização dos assuntos por ordem de importância e a sintetização (Quadro 2) destas que visou à fixação das ideias essenciais para a solução do problema da pesquisa. Quadro 3 a seguir, as variáveis sociodemográficas e clínicas das pacientes.

Quadro 2. Caracterização sociodemográfica de mulheres que realizaram laqueadura, conforme Lima et al (2017)

\begin{tabular}{|r|c|c|c|}
\hline AUTOR & ANO/PUBLICAÇÃ & $\begin{array}{c}\text { IDADE DAS } \\
\text { MULHERES }\end{array}$ & \multicolumn{1}{|c|}{$\begin{array}{c}\text { IDADE/ESCOLARIDADE/ESTADO } \\
\text { CIVIL/RENDA }\end{array}$} \\
\hline $\mathbf{A ~ 0 1}$ & Da Fonseca Bezerra, J. et al 2018 & Não informado & Não informado/Não informado/ \\
\hline $\mathbf{A ~ 0 2}$ & $\begin{array}{c}\text { Brandão, E. R., Pimentel, A. C. } \\
\text { de L., 2020 }\end{array}$ & Não informado & Não informado \\
\hline $\mathbf{A ~ 0 4}$ & Lago, T. Di G. do, et al, 2020 & 20 a 29 & Ensino médio \\
\hline $\mathbf{A ~ 0 5}$ & Lima, I. C. V. de, et al, 2017 & 39 anos & Ensino médio \\
\hline $\mathbf{A ~ 0 6}$ & Sanguinete, T. A. A., 2020 & 31 anos & Não informado \\
\hline
\end{tabular}

Fonte: Autoria própria (2021). 
Quadro 3. Logo abaixo as variáveis sociodemográficas e clínicas das pacientes.

\begin{tabular}{|l|l|}
\hline \multicolumn{2}{|l|}{ Variáveis sociodemográficas e clínicas } \\
\hline Idade & 58 \\
\hline 39 & 44 \\
\hline 40 & 41 \\
\hline Escolaridade & 52 \\
\hline$<9$ & 69 \\
\hline$\geq 10$ & 33 \\
\hline Estado civil & \\
\hline Casada & 68 \\
\hline Solteira/divorciada/separada/viúva & 32 \\
\hline Religião & 20 \\
\hline Católica & \\
\hline Outras (evangélica/espírita/sem religião) & \\
\hline Renda mensal familiar (em salários mínimos)* & \\
\hline$\leq 2$ & \\
\hline$>2$ & \\
\hline
\end{tabular}

\begin{tabular}{|l|l|}
\hline História reprodutiva e uso de métodos contraceptivos \\
\hline Mulher deseja ter filho & \\
\hline Sim & 37 \\
\hline Não & 65 \\
\hline Tem filhos & 86 \\
\hline Sim & 16 \\
\hline Não & \\
\hline Número de filhos & 16 \\
\hline 0 & 35 \\
\hline 1 & 18 \\
\hline 2 & 33 \\
\hline 3 & 24 \\
\hline Laqueadura tubária & 78 \\
\hline Sim & 82 \\
\hline Não & \\
\hline Anticoncepcional oral & \\
\hline Sim & \\
\hline Não & \\
\hline & \\
\hline
\end{tabular}

Fonte: Lima (2017).

Este estudo revelou que mulheres que não receberam informações sobre planejamento familiar são mais propensas a realizar laqueadura tubária, o que pode se associar ao limitado conhecimento e a mitos sobre outros métodos contraceptivos, demonstrando a necessidade dos profissionais de saúde de oferecer aconselhamento às mulheres com Aids sobre opções, benefícios e riscos de cada método, para promover a sua autonomia na tomada de decisão (Lima et al, 2017).

Diante disso, é oportuno analisar a importância da laqueadura, seus riscos e benefícios. A laqueadura, atualmente, é o método contraceptivo mais popular e desejado, sendo considerada a primeira opção para quem procura segurança e eficácia. Porém a irreversibilidade do método pode, inclusive, gerar alguns problemas no relacionamento e afetar o casal emocionalmente (Sanguinete, 2020).

As complicações da laqueadura tubária vêm sendo observadas por alguns autores, porém, outros estudiosos tem negado que existam tais complicações. A respeito das consequências do uso desse método na vida das mulheres, a principal delas que vem sendo cada vez mais abordada é chamada de "síndrome pós laqueadura", tendo destaque para os distúrbios menstruais, e por outro lado, na questão do arrependimento (Lago et al, 2020). 
Research, Society and Development, v. 10, n. 15, e203101523059, 2021

(CC BY 4.0) | ISSN 2525-3409 | DOI: http://dx.doi.org/10.33448/rsd-v10i15.23059

A expressão conhecida como "síndrome pós-laqueadura" que vem sendo empregada para designar um conjunto de sintomas, que aparecem depois da esterilização feminina, caracterizados como desarmonia do ciclo menstrual (metrorragia, sangramento intermenstrual, "spotting" e amenorréia), algia pélvica, dismenorréia, dispareunia, tensão pré-menstrual, mudança no comportamento sexual (diminuição da libido) e manifestações psicológicas. A hipótese mais conhecida a respeito dessa etiologia relacionadas a esses sintomas, apresentada na maioria dos trabalhos sobre o assunto, é a de que eles seriam consequência do dano vascular produzido na cirurgia, provocando o comprometimento ou destruição do suprimento sanguíneo do ovário, alteração da inervação das trompas, torção ovariana e aderências pélvicas (Brandão; Pimentel, 2020).

Além disso, outra consequência destacada na literatura a respeito da laqueadura é o arrependimento. Dentre os fatores de risco que contribuem para o arrependimento pós-laqueadura podem-se destacar a realização do procedimento em mulheres com idade inferior a 25 anos, com informação deficiente sobre o procedimento e outros métodos anticoncepcionais (Oliveira; Rodrigues, 2019).

“A reversão da laqueadura tubária é um processo caro e nem sempre bem-sucedido. Portanto, é importante entender os fatores presentes no momento do pedido de esterilização que possam estar associados com um futuro arrependimento" (Sanguinete 2020, p. 21). Por outro lado, o fator número de filhos são fortes elementos para contribuírem para realização do procedimento, esse mesmo fator também ainda é levantado como um dos principais motivos para gerar o arrependimento na mulher, após a realização da laqueadura, principalmente quanto a morte de um dos filhos ou a mulher se encontra um novo parceiro para o relacionamento, que acende a vontade de se ter outros filhos com o atual parceiro, isso gera inúmeras problemas (Spigolon et al, 2020).

Por outro lado, em relação aos benefícios, a laqueadura tubária ainda vem sendo aquela que apresenta o menor risco de gravidez; acarreta maior economia relacionada aos possíveis gastos com outros anticoncepcionais; não interfere na libido; não interfere no processo de amamentação; pouquíssimas complicações para realização cirúrgica desse procedimento e é benéfico para mulheres que são portadoras de doenças que oferecem risco à saúde (Lima et al, 2017). Abaixo o Quadro 4, com a frequência de mulheres que realizaram laqueadura segundo variáveis sociodemográficas. 
Research, Society and Development, v. 10, n. 15, e203101523059, 2021

(CC BY 4.0) | ISSN 2525-3409 | DOI: http://dx.doi.org/10.33448/rsd-v10i15.23059

Quadro 4. Frequência de mulheres que realizaram laqueadura segundo variáveis sociodemográficas.

\begin{tabular}{|c|c|c|c|}
\hline \multirow{2}{*}{ VARIÁVEIS SOCIODEMOGRÁFICAS } & & \multicolumn{2}{|c|}{ FREQUÊNCIA } \\
\hline & & $\mathrm{N}$ & $\%$ \\
\hline \multicolumn{4}{|l|}{ FAIXA ETÁRIA (ANOS) } \\
\hline & $<25$ & 18 & 6.0 \\
\hline & 25 a 30 & 108 & 35.8 \\
\hline & 31 a 50 & 176 & 58.2 \\
\hline \multicolumn{4}{|l|}{ ZONA } \\
\hline & Rural & 36 & 11.9 \\
\hline & Urbana & 266 & 88.1 \\
\hline \multicolumn{4}{|l|}{ ESTADO CIVIL } \\
\hline & Casada & 248 & 82.7 \\
\hline & Solteira & 52 & 17.3 \\
\hline \multicolumn{4}{|l|}{ NÚMERO DE FILHOS } \\
\hline & 0 & 5 & 1.8 \\
\hline & 1 & 16 & 5.6 \\
\hline & 2 & 120 & 42.3 \\
\hline & 3 & 79 & 27.8 \\
\hline & 4 & 40 & 14.1 \\
\hline & 5 & 16 & 5.6 \\
\hline & 6 & 6 & 2.1 \\
\hline & 7 & 0 & 0.0 \\
\hline & 8 & 2 & 0.7 \\
\hline
\end{tabular}

Fonte: Sanguinete (2020)

Segundo Sanguinete, 2020, “a mediana de idade identificada foi de $31(20,46)$ anos, sendo que 58,2\% das mulheres se encontravam na faixa etária entre 31 à 50 anos, seguida de 38,2\% com idade entre 25 a 30 anos e 6\% com idade menor que 25 anos". No que se refere quanto a moradia, "266 $(88,1 \%)$ moram em zona urbana, e $36(1,9 \%)$ em zona rural, sendo em sua maioria casadas (82,7\%)". Quando analisado o número de filhos foram encontrados 42,3\% com 2 filhos, 27,8\% com 3 filhos e $14,1 \%$ com 4 filhos, foram observadas frequências menores (1,8\%) para aquelas mulheres que não possuíam filhos e $0,7 \%$ para aquelas que possuíam até 8 filhos, evidenciando uma mediana de 3 filhos por mulher. 
A laqueadura foi realizada em 94,7\% pelo convênio SUS, sendo que a realização desse procedimento por plano de saúde e de maneira particular apresentou frequência de $3 \%$ e $2 \%$ respectivamente. No Quadro 5, segue a prevalência dos métodos contraceptivos em mulheres de 14 a 44 anos de idade.

Quadro 5. Prevalência da prática contraceptiva de mulheres com 15 a 44 anos de idade sexualmente ativas * e não grávidas. Município de São Paulo, Brasil, 2015.

\begin{tabular}{|l|c|c|c|}
\hline \multicolumn{1}{|c|}{ PRÁTICA CONTRACEPTIVA } & N & \% & IC95\% \\
\hline Nenhuma & 444 & 15,2 & $13,7-16,8$ \\
\hline Pílula & 760 & 27,0 & $25,2-28,9$ \\
\hline Preservativo masculino & 541 & 19,0 & $17,5-20,6$ \\
\hline Injetáveis & 310 & 10,4 & $9,1-11,8$ \\
\hline Pílula e preservativo masculino & 250 & 8,9 & $7,8-10,1$ \\
\hline Laqueadura & 231 & 7,5 & $6,5-8,7$ \\
\hline Vasectomia & 178 & 6,2 & $5,4-7,2$ \\
\hline Tabelinha e/ou coito interrompido & 81 & 2,8 & $2,2-3,5$ \\
\hline DIU & 73 & 2,5 & $1,8-3,3$ \\
\hline Outras & 17 & 0,6 & $0,4-1,0$ \\
\hline Total & 2.885 & 100,0 & \\
\hline
\end{tabular}

Fonte: Lago et al (2020)

\section{Discussão}

A esterilização tubária consiste num procedimento cirúrgico relativamente novo. Assim, esse procedimento e/ou método funciona no controle da fertilidade, os estudos apontam que essa é uma técnica do século XX. Todavia, suas origens mais profundas encontram-se pautadas no século XIX, especificamente no ano de 1809, quando Haighton construiu alguns experimentos seccionando as tubas de coelhas. Em procedimentos realizados em humanos, Blundell, em Londres, no ano de 1823, é visto pelos ingleses como o pioneiro quanto se trata da ligadura tubária (Spigolon, 2020).

Todavia, como não se pode encontrar uma publicação específica na literatura médica como prova do fato mencionado anteriormente, outros autores, mais precisamente os americanos, consideram que foi Lungren (1881), nos Estados Unidos, o primeiro a executar o procedimento da ligadura tubária. A experiência realizada por ele através da esterilização tubária, fez o uso de fios e seda para segurar as tubas de uma paciente que já tinha feito duas operações cesarianas (Spigolon, 2020).

Logo no começo do século XX, a esterilização cirúrgica ganhou mais espaço e sua prática acabou se tornando algo rotineiro e comum, e na maioria das vezes por motivos eugênicos, entre eles, o retardo mental severo. A partir do ano de 1930, com algumas evoluções da área da clínica cirúrgica e com o advento das sulfonamidas e da penicilina, o uso desta operação passou a ser alcançar uma dimensão ainda maior. Entretanto, sua história sempre seguida tendo relação direta ao Movimento Eugênico até que, por causa dos abusos do nazismo, a prática começou a receber alguns questionamentos e por um período foi deixada de lado (Sanguinete, 2020).

A volta procedimento cirúrgico veio a ocorrer nos anos 60, quando existiu um grande interesse pela esterilização voluntária, especialmente quando se tratava das questões populacionais, mas não se pode negar que diante da introdução de 
novas tecnologias, como a laparoscopia, foram surgindo novas técnicas com procedimentos mais simples e mais efetivas (Lago, et al, 2020).

Por volta dos anos 70, mais precisamente no início, essa técnica cirúrgica acabou se fortalecendo mais como prática contraceptiva mais adequada, com a chegada dos anos 90, esse foi o método de contracepção mais realizado no mundo. No ano de 1981, por exemplo, Stepan realizou alguns cálculos que o direcionaram para o resultado de 260 milhões de casais espalhados pelo mundo que já haviam se submetido a algum tipo de esterilização (Brandão; Pimentel, 2019; dos Santos et al, 2020).

Nos países de Terceiro Mundo, por exemplo, é constatado uma clara tendência em favor da esterilização como a melhor opção contraceptiva (Soares, 2019). Este fenômeno não foi constatado apenas nos países do Terceiro Mundo. Nos Estados Unidos, o número de esterilizações apresentou grande crescimento. Em 1977, Hulka, da Associação para a Esterilização Voluntária, calculava que mais de oito milhões de homens e mulheres na América do Norte haviam sido esterilizados cirurgicamente (Lima et al, 2017).

No Brasil, já faz alguns anos que se tem identificado um aumento bem consistente da prevalência da esterilização feminina, comumente a laqueadura tubária, sendo caracterizada na atualidade como a prática contraceptiva mais realizada (Theisen, 2020). Os métodos cirúrgicos são métodos de contracepção definitiva que podem ser utilizadas tanto pelas mulheres, como pelos homens, promovendo a esterilização. No caso das mulheres é realizado por meio da ligadura das trompas, e nos homens por meio da ligadura dos canais deferentes - vasectomia (Spigolon, 2020; Medeiros, 2019).

Laqueadura é um procedimento de que leva à esterilização feminina. A anticoncepção definitiva ocorre pelas trompas, estrutura em que ocorre o encontro do óvulo com os espermatozoides (fecundação). A obstrução deste canal inviabiliza a fecundação ou a fertilização, impossibilitando a mulher de engravidar por esta via. Atualmente, existe um "stent tubário" (semelhante a uma mola) que é colocado por histeroscopia ambulatorial, sem anestesia com a mesma função de obstruir a trompa (Sanguinete, 2020).

Por se tratar um método de caráter definitivo, é necessário que haja um aconselhamento esclarecido sobre a eficácia, segurança, as vantagens e as desvantagens desse tipo de método, desencorajando essa escolha, pois é irreversível (Lago et al, 2020). Existem diversas técnicas de promover a ligadura das tubas uterinas, no entanto, a mais utilizada é a salpingectomia segmentar. Em relação ao acesso cirúrgico às trompas, esse mesmo autor afirmar que podem ser feitos de diversas maneiras, dentre elas destacam-se a minilaparotomia, que consiste numa pequena incisão suprapúbica, com 4 a $5 \mathrm{~cm}$ de tamanho; a laparotomia; a laparoscopia; a culdotomia, que consiste no acesso feito pela abertura da vagina; e a histeroscopia, onde busca os óstios tubários que serão submetidos à eletrocoagulação (Lima et al, 2017).

Os métodos definitivos alta eficácia, principalmente no primeiro ano, onde a taxa de gravidez é de 0,5 a cada 100 mulheres. É importante ressaltar que para esse método obtenha essa alta eficácia, é necessário levar em consideração a técnica cirúrgica utilizada, o tempo da cirurgia e a idade da paciente (Brandão; Pimentel, 2019).

Por exemplo, nos estudos realizados por Lago et al (2020, p. 11):

Por outro lado, entre as mulheres com 3 ou mais filhos, a laqueadura foi a prática mais frequente, embora tenha sido superada pelo uso de hormonais injetáveis no grupo etário de 20 a 29 anos. Entre as mulheres com 30 a 44 anos, a maior escolaridade se associou a um mix no qual a laqueadura permaneceu mais frequente, porém com maior participação relativa do preservativo masculino e da vasectomia. 
Dando continuidade aos estudos de Lago et al (2020), é possível dizer que o perfil de práticas contraceptivas observado indica o menor recurso à esterilização feminina, isso transparece na preocupação de uma parcela das mulheres com a dupla proteção, que o uso dos hormonais injetáveis acabou se tornando mais frequente e que aumentou a participação masculina. No entanto, o DIU, método altamente eficaz e que pode se constituir na única alternativa de LARC não hormonal, praticamente quase não vem sendo utilizado, provavelmente por causa da pouca oferta.

Por outro lado, Brandão e Pimentel (2020, p. 9) destacam que a dificuldade para a laqueadura tubária envolve outros segmentos:

As dificuldades existentes no âmbito do SUS para acesso à contracepção e obtenção da laqueadura tubária, com grande demanda de mulheres que guardam vagas/leitos para tal procedimento eletivo, pode ter sido um cenário propício para oferta de um método novo, menos invasivo, realizado em ambulatórios e que prometia resolver definitivamente as angústias femininas com o risco de uma gravidez imprevista.

Com base nos estudos de Lima et al (2027), este estudo revelou que mulheres que não receberam informações sobre planejamento familiar são mais propensas a realizar laqueadura tubária, o que pode se associar ao limitado conhecimento e a mitos sobre outros métodos contraceptivos, demonstrando a necessidade dos profissionais de saúde de oferecer aconselhamento às mulheres com Aids sobre opções, benefícios e riscos de cada método, para promover a sua autonomia na tomada de decisão.

Por fim, nos estudos realizados por Spigolon (2020), as puérperas que tiveram o parto cesárea por causa da falha na obtenção de dilatação ou mesmo com vistas à realização da laqueadura apresentaram satisfação com a escolha, pois para algumas delas não mais haveria a dor e um método para não engravidar novamente. Entretanto, segundo Vieira, (2020), entre as que não se sentiram satisfeitas, deveu-se à não realização de laqueadura e/ou histerectomia, ou pela insatisfação da cicatriz do parto. Logo, a maior parte das puérperas ficaram satisfeitas com a via de parto escolhida e realizada.

\section{Conclusão}

É importante sempre reforçar a necessidade das mulheres, de se informar sobre os procedimentos cirúrgicos que desejam se submeter: seja à laqueadura, seja a tratamentos na área das novas tecnologias reprodutivas, ela precisa ser consciente, principalmente das consequências pós procedimentos. $\mathrm{O}$ acesso às informações resulta numa melhor familiaridade com os termos e mais segurança ante as escolhas além de minimizar as chances de arrependimento após o procedimento.

As ações de aconselhamento, orientação, avaliação clínica e acompanhamento de saúde não podem deixar de ser executados, para oferecer o método contraceptivo apropriado a cada mulher, em cada fase de seu ciclo de vida e em condições específicas de parceria sexual (ocasional, estável, múltiplas) adequando essa escolha para cada contexto cultural particular. A laqueadura tubária foi discutida nesse trabalho de forma mais aprofundada com base em seis estudos científicos.

Entre as mulheres com 3 ou mais filhos, a laqueadura foi a prática mais frequente, embora tenha sido superada pelo uso de hormonais injetáveis no grupo etário de 20 a 29 anos. Essa realidade evidencia a necessidade de implantação de medidas de educação em saúde bem como de estratégias focadas nesse público, pois arrependimentos e consequências fazem parte desse processo, como foram pontuadas nos estudos selecionados.

Portanto, essa pesquisa abriu novas possibilidades para aprofundar os conhecimentos a respeito da laqueadura tubária e das variáveis ligadas as mulheres que escolhem esse procedimento. No entanto, há necessidade de novos estudo sobre a temática, no intuito de trazer novas abordagens, a fim de promover um melhor esclarecimento sobre o assunto. 
Research, Society and Development, v. 10, n. 15, e203101523059, 2021

(CC BY 4.0) | ISSN 2525-3409 | DOI: http://dx.doi.org/10.33448/rsd-v10i15.23059

\section{Referências}

Brandão, E. R., \& Pimentel, A. C. D. L. (2019). Essure no Brasil: desvendando sentidos e usos sociais de um dispositivo biomédico que prometia esterilizar mulheres. Saúde e Sociedade, 29, e200016.

BrasiL. Ministério da Saúde. (2013). Secretaria de Atenção à Saúde. Departamento de Atenção Básica. Saúde sexual e saúde reprodutiva / Ministério da Saúde, Secretaria de Atenção à Saúde, Departamento de Atenção Básica. n. 26. Brasília: Ministério da Saúde.

Bruner, J. (2002). Atos de significação. 2. ed. Trad. Sandra Costa. São Paulo: Artmed, 2002.

da Fonseca Bezerra, J., de Lara, S. R. G., do Nascimento, J. L., \& Barbieri, M. (2018). Assistência à mulher frente à violência sexual e políticas públicas de saúde: revisão integrativa. Revista Brasileira em Promoção da Saúde, 31(1), 1-12.

de Lima, I. C. V., dos Santos Oliveira, M. D. C., da Cunha, G. H., \& Galvão, M. T. G. (2017). Aspectos reprodutivos e conhecimento sobre planejamento familiar de mulheres com Síndrome da Imunodeficiência Adquirida. Revista da Escola de Enfermagem da USP, 51 , e03224.

dos Santos, R. L., de Oliveira Barbosa, A. D. L., Santana, A. L., Farias, J. V. C., de Macêdo, P. R., \& Farias, I. C. C. (2020). Os riscos do uso prolongado de contraceptivos hormonais. Research, Society and Development, 9(11), e69791110394-e69791110394.

Fernandes, T. C. (2020). Direito, restrições e violações da laqueadura no brasil. https://repositorio.ivc.br/handle/123456789/281

Gil, A. C. (2008). Como elaborar projetos de pesquisa. 4. ed. São Paulo: Atlas, 2008.

Gonçalves, T. A. (2017). Direitos humanos das mulheres e a Comissão Interamericana de Direitos Humanos. Saraiva Educação SA.

Lago, T. D. G. D., Kalckmann, S., Alves, M. C. G. P., Escuder, M. M. L., Koyama, M., \& Barbosa, R. M. (2020). Diferenciais da prática contraceptiva no Município de São Paulo, Brasil: resultados do inquérito populacional Ouvindo Mulheres. Cadernos de Saúde Pública, 36.

Lakatos, E. M., \& Marconi, M. de A. (2010). Fundamentos da metodologia científica. 7. ed. São Paulo: Atlas, 2010.

Marcondes, M. M. (2021). Políticas públicas para e pelas mulheres. http://revistaconstrucao.org/politica-de-genero/politicas-publicas-para-e-pelas-mulheres/.

Medeiros, A. P. P. de, Santana, C. M., Ferreira, D. O., Ribeiro, K. C., \& Brito, M. L. de A. (2019). A Importância do Planejamento Gestacional para Diferentes Gerações de Mulheres. Research, Society and Development, 8(2), e2282661. https://doi.org/10.33448/rsd-v8i2.661

Oliveira, A. M., \& Rodrigues, H. W. (2019). Blessed be the fruit: resquícios de um viés controlista em ações sobre cirurgia de laqueadura no Judiciário de Santa Catarina (2015-2016). Revista Direito GV, 15.

Sanguinete, T. A. A. (2020). Perfil das mulheres que realizaram laqueadura em uma maternidade de referência do Alto do Jequitinhonha - MG. Dissertação. Diamantina, 2020.

Soares, L. P. (2019). Esterilização da mulher e direitos reprodutivos: análise das políticas públicas de contracepção à luz da Lei no 9.263/96 diante da autonomia do corpo.

Spigolon, D. N., Teston, E. F., Maran, E., Varela, P. L. R., Biazyan, S. E., Ribeiro, B. M. dos S. (2020). Percepções das gestantes quanto à escolha da via de parto. Saúde e Pesquisa. 13(4): 789-798.

Theisen, C. (2020). Esterilização cirúrgica na região de Saúde Fronteira Noroeste como forma de Política Pública para Planejamento Familiar. https://rd.uffs.edu.br/handle/prefix/4141

Teodoro, L. P. P., Torres, G. M. C., da Silva Filho, J. A., Figueiredo, I. D. T., Cândido, J. A. B., da Silva Quirino, G., ... \& Pinto, A. G. A. (2020). Percepções de usuárias sobre as ações de enfermagem para saúde sexual e reprodutiva. Research, Society and Development, 9(12), e1891210409-e1891210409.

Vieira, S. (2021). Esterilização Voluntária e a Autonomia Reprodutiva da Mulher Casada. Editora Dialética. 